5. E. C. Zeeman, Unknotting spheres in five dimensions, Bull. Amer. Math. Soc. 60 (1960), 198.

6. - Unknotting spheres, Ann. of Math. (2) 72 (1960), 350-361.

7. - Unknotting combinatorial balls, (to appear).

8. - Isotopies of manifolds, (to appear).

Florida State University and

Gonville and Caius College, Cambridge

\title{
A WEAK TYCHONOFF THEOREM AND THE AXIOM OF CHOICE
}

L. E. WARD, JR. ${ }^{1}$

1. A well-known result of Kelley [1] asserts that the Tychonoff theorem (the product of compact spaces is compact) implies the axiom of choice, establishing the equivalence of these propositions. In what follows we show that an apparently weaker form of the Tychonoff theorem also implies the axiom of choice. The proof is quite brief and direct.

WeaK TychonofF Theorem. The product of a family of mutually homeomorphic compact spaces is compact.

Proposition. The weak Tychonoff theorem implies the axiom of choice.

Proof. Let $a$ be a disjoint family of nonempty sets and let $\Sigma$ be the union of the members of $a$. If $\Sigma^{a}$ denotes the set of functions on $a$ into $\Sigma$, we shall demonstrate the existence of an element of $\Sigma a$ which is a choice function, i.e., a function which maps each $A$ in $a$ into an element of itself.

Topologize $\Sigma$ by defining a subset $U$ of $\Sigma$ to be open if $U$ is the empty set or if $\Sigma-U$ is the union of finitely many members of $a$. Since $a$ is a disjoint family this collection of open sets clearly satisfies the axioms for a topology, and it is easily seen to be a compact topology. By the weak Tychonoff theorem, $\Sigma^{Q}$ is also compact.

For each $A \in Q$ let $F_{A}$ denote the set of all $f \in \Sigma^{Q}$ such that $f(A)$

Received by the editors September 12, 1961.

1 This research was supported by the United States Air Force through the Air Force office of Scientific Research of the Air Research and Development Command, under Contract No. AF 49(638)-889. Reproduction in whole or in part is permitted for any purpose of the United States Government. 
$\in A$. Note that $F_{A}$ is simply the inverse image of $A$ under the $A$ th projection mapping of $\Sigma Q^{a}$ onto $\Sigma$, and therefore $F_{A}$ is a closed set. We assert that the collection of all $F_{A}$ has the finite intersection property. For if $A_{1}, A_{2}, \cdots, A_{n}$ are members of $a$ then (by the finite axiom of choice) there is a function $g$ defined on $\left\{A_{1}, A_{2}, \cdots, A_{n}\right\}$ such that $g\left(A_{i}\right) \in A_{i}$ for each $i=1,2, \cdots, n$. If we extend $g$ to $a$ by defining $g(A)=g\left(A_{1}\right)$ for each $A \in Q, A \neq A_{i}$, then $g \in F_{A_{1}} \cap \cdots \cap F_{A_{n}}$. Since

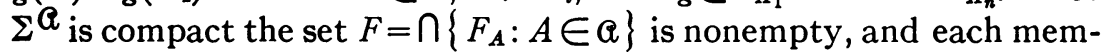
ber of $F$ is a choice function. The proof is complete.

It should be remarked ${ }^{2}$ that we have actually proved a slightly deeper proposition than that asserted. It can be stated as follows:

TheOREM. Let a be a disjoint family of nonempty sets covering the set $\Sigma$, and topologize $\Sigma$ by using $a$ as a subbase for the closed sets. If $\Sigma^{Q}$ is compact then there is a choice function for $Q$.

2. Since the axiom of choice implies the Tychonoff theorem, it follows that the weak Tychonoff theorem implies it as well. It is interesting that this fact can be proved directly without employing the choice axiom. ${ }^{3}$ To see this, suppose $S$ is a family of compact spaces. We may, without loss of generality, assume $S$ to be a disjoint family. Let $M$ denote the union of the members of $S$, and topologize $M$ by defining the subset $U$ of $M$ to be open if $U$ is the empty set or if $U$ contains all but a finite number of members, $S_{1}, S_{2}, \cdots, S_{n}$, of $\delta$, and $U \cap S_{i}$ is open in $S_{i}$ for each $i=1,2, \cdots, n$. It is clear that $M$ is compact with respect to this topology and hence, by the weak Tychonoff theorem, $M^{\mathcal{S}}$ is compact. If $\pi_{S}: M^{\mathcal{S}} \rightarrow M$ is the $S$ th projection mapping then $\pi_{S}^{-1}(S)$ is closed in $M^{\mathcal{S}}$ and hence $S_{0}=\bigcap\left\{\pi_{S}^{-1}(S): S \in \mathcal{S}\right\}$ is closed. But it is easy to see that $S_{0}$ is precisely the product of the family $S$, and since $S_{0}$ is a closed subset of a compact space, it is compact.

\section{REFERENCE}

1. J. L. Kelley, The Tychonoff product theorem implies the axiom of choice, Fund. Math. 37 (1950), 75-76.

\section{The University of Oregon}

${ }^{2}$ I am grateful to the referee for this observation.

3 I am indebted to F. W. Anderson for a portion of this proof. 